\author{
Alberto Sicilia \\ Marc Quirynen \\ Alain Fontolliet \\ Helena Francisco \\ Anton Friedman \\ Tomas Linkevicius \\ Rainer Lutz \\ Henny I. Meijer \\ Eric Rompen \\ Roberto Rotundo \\ Frank Schwarz \\ Massimo Simion \\ Wim Teughels \\ Ann Wennerberg \\ Otto Zuhr
}

\section{Long-term stability of peri-implant tissues after bone or soft tissue augmentation. Effect of zirconia or titanium abutments on peri-implant soft tissues. Summary and consensus statements. The 4th EAO Consensus Conference 2015}

Authors' affiliations:

Alberto Sicilia, University of Oviedo, Oviedo, Spain Marc Quirynen, Wim Teughels, Catholic

University of Leuven, Leuven University Hospital, Leuven, Belgium

Alain Fontolliet, University of Zurich, Zurich, Switzerland

Helena Francisco, University of Lisbon, Lisbon, Portugal

Anton Friedman, Witten-Herdecke University,

Witten, Germany

Tomas Linkevicius, University of Vilnius, Vilnius, Lithuania

Rainer Lutz, University of Erlangen-Nuremberg, Erlangen, Germany

Henny J. Meijer, University of Groningen,

Groningen, Netherlands

Eric Rompen, University of Liège, Liège, Belgium

Roberto Rotundo, University of Florence, Florence, Italy

Frank Schwarz, Heinrich Heine University,

Düsseldorf, Germany

Massimo Simion, University of Milan, Milan, Italy

Ann Wennerberg, University of Malmö, Malmö,

Sweden

Otto Zuhr, Private Practice in Munich, University of Frankfurt, Frankfurt, Germany

*Corresponding author:

Alberto Sicilia

University of Oviedo. Faculty of Medicine and

Health Sciences

University Clinic of Dentistry, Section of

Periodontology

Catedrático José Serrano sn. 33006 Oviedo, Spain

e-mail: asicilia@clinicasicilia.es

Date:

Accepted 18 May 2015

To cite this article:

Sicilia A, Quirynen M, Fontolliet A, Francisco H, Friedman A, Linkevicius T, Lutz R, Meijer HJ, Rompen E, Rotundo R, Schwarz F, Simion M, Teughels W, Wennerberg A, Zuhr O. Long-term stability of peri-implant tissues after bone or soft tissue augmentation. Effect of zirconia or titanium abutments on peri-implant soft tissues. Summary and consensus statements. The 4th EAO Consensus Conference 2015. Clin. Oral Impl. Res. 26 (Suppl. 11), 2015, 148-152

doi: $10.1111 /$ clr.12676

Key words: hard tissue augmentation, peri-implant tissues, soft tissue augmentation, titanium abutment, Zirconia abutment

\begin{abstract}
Introduction: Several surgical techniques and prosthetic devices have been developed in the last decades, aiming to improve aesthetic, hygienic and functional outcomes that may affect the periimplant tissues, such as procedures of bone and soft tissue augmentation and the use of custommade abutments of titanium and zirconium.

Materials and methods: Three systematic reviews, based on randomized clinical trials and prospective studies covering the above reported topics were analysed, and the detected evidence was exposed to interactive experts' discussion during the group's and general assembly's meetings of the 4th EAO Consensus Conference. The results are reported using the following abbreviations: S$\mathrm{T}$ : short-term evidence, M-T: medium-term evidence; L-T: long-term evidence; LE: limited evidence. Results: Soft tissue augmentation procedures may be indicated for the increase of soft tissue thickness and keratinized tissue, the reduction of interproximal peri-implant bone loss, and the coverage of shallow peri-implant soft tissue recessions (S-T, LE), L-T is lacking. Guided bone regeneration approaches (GBR) showed efficacy when used for ridge reconstruction after the complete healing of the soft tissues (S-T \& L-T), and the stability of the augmented bone may play a role in the maintenance of the soft tissue position and dimensions (LE). No significant differences were observed between titanium and zirconia abutments when evaluating probing pocket depth, bleeding on probing, marginal bone levels and mucosal recessions. Zirconia abutments were associated with more biological complications but demonstrated superiority in terms of achieving natural soft tissue colour (S-T).
\end{abstract}

The task of this working group was to analyse the effect of soft or hard tissue augmentation on the peri-implant tissues, including the long-term stability of the observed results, as well as to analyse the effect on the peri-implant tissues of the use of zirconia and titanium abutments.

Three systematic reviews were evaluated, and the findings reported were exposed to interactive experts' discussion within the group and general assembly meetings of the 4th European Association for Osseointegration Consensus Conference (4th EAO$\mathrm{CC)}$ :

Long-term outcomes of soft tissue augmentation around dental implants on soft and hard tissue stability. A systematic review. (Rotundo et al. 2015) 
Long-term outcomes of bone augmentation on soft and hard tissue stability. A systematic review. (Lutz et al. 2015)

The effect of zirconia or titanium as abutment material on soft tissues. A systematic review and meta-analysis. (Linkevicius \& Vaitelis 2015)

Using this methodology, three consensus report were elaborated containing the summaries of the reviews, which included the group's analysis of the information obtained from the tables and references in each review, as well analysis of additional references discovered during the group's discussion. Along with this condensed information, a translational effort was made, including experts' opinions on clinical criteria, to elaborate the "consensus statements", "clinical recommendations" and "implications for research".

Long-term outcomes of soft tissue augmentation around dental implants on soft and hard tissue stability. A systematic review

\section{Aim}

To investigate the long-term outcomes, in terms of peri-implant soft tissue height and thickness, on bone levels, when soft tissue augmentation has been performed.

\section{Summary of the review}

There is no long-term evidence to suggest that soft tissue augmentation may have an effect on peri-implant soft tissue thickness and keratinized tissue width (KTW) or on the stability of peri-implant bone levels.

The available results, summarized below, are limited to short and medium-term evaluation and are based on 10 papers, mainly with a 1 -year follow up period. Two studies reported medium-term data (4-9 years), both at high risk of bias (Bianchi \& Sanfilippo 2004; Bruschi et al. 2014). Eight of them reported on soft tissue augmentation performed at the time of implant placement or at the second stage surgery. The other two dealt with treatment of peri-implant soft tissue dehiscences after prosthetic rehabilitation (both with 1 year of follow up). These two papers were eligible and used for meta-analysis.

Effect of soft tissue augmentation on soft tissue thickness and keratinized tissue width

Data from two studies were analysed. The first of these was a randomized clinical trial (RCT) evaluating the use of a connective tissue graft (CTG) during the first stage of implant place- ment in 10 patients (Wiesner et al. 2010). The second was a prospective case series study (PCS) evaluating the treatment of peri-implant recession coverage in 20 patients (Zucchelli et al. 2012). The data from these studies suggested that the use of a CTG is related with an increase of soft tissue thickness of between 1.2 and $1.6 \mathrm{~mm}$ at 1 year.

It is difficult to summarize data on KTW from the available information due to the heterogeneity of the procedures. In cases of soft tissue augmentation performed at the time of implant placement, a PCS study (split-thickness apically repositioned flaps ARF- with 85 patients at 4 years) (Bruschi et al. 2014) reported an average increase of $5.1 \mathrm{~mm}$. In cases of soft tissue augmentation performed after the prosthetic rehabilitation, a RCT at 1 year (free gingival graft or vestibuloplasty in 64 patients) (Basegmez et al. 2012) reported an increase of KTW between 1.2 and $2.4 \mathrm{~mm}$. In cases where soft tissue dehiscences were present, a PCS study (CAF+CTG on 20 patients at 1 year) (Zucchelli et al. 2012), showed an increase of KTW of $0.6 \mathrm{~mm}$.

\section{Effect of soft tissue augmentation on peri-implant} bone levels

Three studies were identified which used two different approaches:

Autogenous connective tissue applied buccally: Data from 1 RCT (CTG on the buccal side, or not, at the first stage of implant placement - 10 patients/split mouth) showed no differences between connective tissue grafted and non-grafted areas in terms of mean marginal bone levels (mMBLs) at 1 year (Wiesner et al. 2010).

Allogenic connective tissue substitutes (ACTS) applied over the crest: Two PCTs (ACTS placed over the crest at implant placement, or not), which involved in total 144 patients, reported significantly lower interproximal marginal peri-implant bone resorption in the grafted areas at 1 year (about 0.2 vs. $1.5 \mathrm{~mm}$ ) (Linkevicius et al. 2013; Puisys \& Linkevicius 2013).

\section{Effect of soft tissue augmentation on implant/ abutment coverage}

Data from two PCSs at 1 year (Zucchelli et al. 2012; Roccuzzo et al. 2013) involving the treatment of shallow peri-implant soft tissue dehiscences by means of $\mathrm{CAF}+\mathrm{CTG}$, showed positive results, reporting a complete defect coverage in $56 \%$ to $75 \%$ of the cases. The related Bayesian meta-analysis indicated a mean implant/abutment coverage of $1.7 \pm 0.01 \mathrm{~mm}$ (CrI:1.4;1.9).
Effect of soft tissue augmentation on aesthetic evaluation

Only one study /CS of 20 patients on abutment coverage) reported data on aesthetic evaluation from the patient's point of view, showing that the patients perceived a significant aesthetic improvement up to 1 year after the procedure (Zucchelli et al. 2012).

Data from two studies with 1-year followup (1 RCT on implant placement +CTG in the posterior area, and 1 PCS on implant/ abutment coverage in the anterior area) evaluating the aesthetic results from the practitioner's point of view showed a significant aesthetic improvement after both treatments (Wiesner et al. 2010; Roccuzzo et al. 2013).

\section{Group's consensus}

At short and medium-time observation periods there exists limited evidence that:

1. Soft tissue augmentation procedures are able to increase the peri-implant soft tissue thickness and the width of keratinized tissue.

2. In posterior mandibular areas with thin crestal soft tissue, marginal peri-implant bone loss may be reduced by means of vertical soft tissue thickening procedures (ACTS).

3. The coverage of exposed implant/abutment surfaces related to shallow marginal soft tissue recessions may be achieved by means of CAF+CTG.

4. Subjective and objective aesthetic results may be improved by means of soft tissue augmentation procedures.

However, due to the lack of studies, there is no long-term evidence that the soft tissue augmentation may have an effect on peri-implant soft tissue thickness, keratinized tissue width, soft tissue marginal levels and on the stability of peri-implant bone levels.

\section{Clinical recommendations}

The group's opinion, based on limited data, is that soft tissue augmentation procedures may be performed to increase the peri-implant soft tissue thickness, the width of keratinized tissue, and to reduce marginal peri-implant bone loss in areas with thin soft tissues.

In the presence of peri-implant soft tissue dehiscences, it may be possible to cover the exposed implant/abutment surface by means of a CAF+CTG.

So far, long-term evidence is too limited to strongly recommend peri-implant soft tissue augmentation procedures. The decision must at this stage be left to clinician judgement after a thorough patient evaluation. 


\section{Implications for research}

There is a need for studies evaluating, at least over a medium and long-term period (5-10 or more years), the repercussions of peri-implant soft tissue augmentation on the stability of the soft and hard peri-implant tissues, as well as the aesthetic aspects and patient-related outcome measures.

Future studies must include the following clinical and radiological evaluations as a minimum:

1. Assessment of peri-implant tissue health.

2. Peri-implant keratinized tissue width changes (KTW).

3. Peri-implant marginal soft tissue level changes (PSL).

4. Peri-implant soft tissue thickness changes (KTT).

5. Peri-implant marginal bone level changes (MBL).

6. Professional aesthetic evaluation.

7. Patient reported outcome measures (PROMS).

New validated technologies should be used to further improve the accuracy and reproducibility of the measurements.

\section{Long-term outcomes of bone augmentation on soft and hard- tissue stability. A systematic review}

\begin{abstract}
Aim
This review analysed the current literature regarding medium- and long-term data concerning the stability of peri-implant tissues after hard-tissue augmentation, prior to or at the same time as implant placement.
\end{abstract}

\section{Summary of the review}

Twenty-seven prospective studies were identified, dealing with guided bone regeneration procedures (21), socket grafting (4) and bone stability around implants that were placed in previously augmented bone (2). Only six were randomized clinical trials (RCTs) that were designed to compare different augmentation techniques. These had a follow-up of between 1 and 5 years. One RCT included a negative control group evaluating bone changes around implants placed into pristine alveolar bone. The studies evaluated bone regeneration associated with implant placement in three different scenarios: guided bone regeneration plus delayed implant placement, guided bone regeneration plus simultaneous implant placement, and socket preservation with graft simultaneous to implant placement.
Several prospective studies considered bone augmentation using different techniques. The follow-up period ranged from 1 to 12.5 years.

Twenty-three studies evaluated two-dimensional interproximal bone level changes by calculating mean marginal bone loss by means of peri-apical radiographs (5 RCTs and 18 PCTs). They included 725 patients and 1358 implants. Only six studies evaluated the bone levels three-dimensionally by means of a cone beam computer tomographic examination (CBCT). These were made up of two RCTs and four controlled clinical trials (CCTs). All of them dealt with different modalities of guided bone regeneration (GBR) associated with dental implant treatments. They evaluated 141 patients and 188 implants, with follow-up periods ranging from 1 to 7 years.

Thirteen studies evaluated soft tissue levels by measuring soft tissue recession using either a periodontal probe or calibrated photographs. These included 518 patients and 849 implants. Two studies used a radiopaque marker to evaluate soft tissue dimensions in CBCT scans (46 patients and 46 implants).

Stability of augmented bone

After an augmentation procedure the characteristics of the regenerated tissue depend on the materials used within the graft (among other factors). Hence, it cannot always be considered as vital bone. However, to be consistent with the current literature the words "bone", "bone levels", "bone gain" and similar terms, are used in the present consensus report.

GBR procedures for bone reconstruction, performed simultaneously with the placement of dental implants, in situations in which adequate soft tissue healing was accomplished before the surgery, obtained significant bone gain (as evaluated by means as a CBCT examination) and interproximal bone levels were reported as being stable over the medium-term period. However, no comparison was available between CBCTs taken at different time points after the implant placement (Jung et al. 2015; Buser et al. 2013a, 2013b; Miyamoto et al. 2011).

The group was, however, able to make the general observation that following the use of GBR techniques, marginal bone levels (mMBL), assessed by means of periapical radiographs, seemed to be stable over 112.5 years, with bone loss ranging from 0.1 to $0.8 \mathrm{~mm}$ (Mejndert et al. 2007; Merli et al. 2010; Buser et al. 2013a; Amorfini et al. 2013; Jung et al. 2013).
Only one study (CCT) examined the use of GBR techniques simultaneously with implant placement, immediately after tooth extraction. This reported that at 7 years 36\% of the implants treated showed almost a nondetectable buccal plate (Benic et al. 2012).

There is a lack of data in the present review about the stability of the augmented bone using surgical techniques other then GBR, mainly because the type of studies available were generally case reports or retrospective studies. Only two prospective clinical studies were detected. These dealt with the use of autogenous bone, particulated or blocks, associated to DBBM and fibrin glue, or to a titanium mesh, with a follow-up of 2 and 3 years. They evaluated bone loss and demonstrated a mean marginal bone reduction of between 0.3 and $1.1 \mathrm{~mm}$ (Von Arx et al. 1998; Hellem et al. 2003).

Effect of bone augmentation on soft tissue stability Long-term soft tissue stability was difficult to evaluate with the information retrieved from the reviewed papers, as the baseline reference point and measurements were unclear and not applied consistently in many studies. However, limited data exist to evaluate whether following GBR the soft tissue profile is maintained over time.

Two studies (PCTs), dealing with GBR procedures simultaneous to early implant placement, clinically evaluated the distance between the implant shoulder of bone-level implants and the mucosal margin (DIM) at two time points with a 4 and 5 years interval, showing that DIM increased with time. The increment ranged from 0.3 to $0.4 \mathrm{~mm}$, indicating stability or soft tissue coverage of the implant-supported crown (Buser et al. 2013a, 2013b).

A few studies show a relationship between the position of the soft tissue margins and the level of the peri-implant bone (Benic et al. 2012; Miyamoto et al. 2011; Dahlin et al. 2010).

\section{Consensus statements}

GBR procedures have been shown to be effective in augmenting the alveolar bone - when performed after completion of soft tissue healing. The regenerated bone seems to be stable over time. There is limited evidence to indicate that the remodelling pattern is similar to that of native bone.

Stability of the augmented bone was also reported, in the short-term, with techniques using autogenous bone, with or without DBBM, and titanium mesh or fibrin glue acting as stabilizers of the graft. 
GBR performed at the time of tooth extraction together with immediate implant placement has been associated with significant loss of the buccal bone plate.

Limited information exists regarding the relation between the stability of the soft tissue margin and that of the regenerated bone, even though a small number of studies may indicate a positive relationship between the underlying bone level and the soft tissue margin position.

\section{Clinical recommendations}

When aiming at early implant placement, i.e. 4-8 weeks after the tooth extraction, or at the placement of an implant in a healed ridge performing a simultaneous hard tissue augmentation, GBR is a reliable method to reconstruct the ridge and ends-up with increased and stable bone volume.

However, ridge augmentation with GBR techniques associated to immediate implants performed immediately after tooth extraction may not be recommendable, as further loss of the buccal plate may occur.

Consistent bone ridge reconstructions obtained by means of GBR procedures may facilitate the maintenance of a stable buccal soft tissue contour.

Based on the limited evidence and on experts' opinions, the group consents that residual marginal defects which lead to the exposure of the implant surface must be avoided by clinicians when using moderately rough surfaced implants in combination with GBR procedures, since these areas may be prone to the development of peri-implant diseases on the long-term (Schwarz et al. 2012; Schwarz et al. 2014; Fontana et al. 2015).

There is a lack of information about threedimensional hard and soft tissue evolution following the use of other kinds of hard tissue augmentation techniques.

\section{Implications for research}

The great variation in the way outcomes are reported, the lack of consistency between the methods of evaluation during follow-up, as well as the difficulty of establishing adequate baseline reference points, makes it very difficult to summarize data and make comparisons among different studies.

Additionally, the outcome of bone regeneration procedures should be evaluated in all three dimensions. Therefore, further research should concentrate on a more precise evaluation of hard and soft tissue reconstruction by means of a three-dimension analysis, either using CBCT (respecting EURATOM regulations) or using alternative approaches based on intraoral or lab-based optical scanner technology, or ultrasonic devices.

Although short-term studies (1 year) provide very valuable information of the treatment outcomes, to assess hard and soft tissue stability medium and long-term evaluation is recommended (more than 5 years).

The impact of implant characteristics on the long-term outcome of bone augmentation has still to be explored.

\section{Measurements for bone loss evaluation} Mean marginal bone loss (mMBL) measured on periapical radiographs is the most frequently used method to assess interproximal bone loss. Using this technique the distance from the implant shoulder to the bone to implant contact point can be identified. However, no information about the buccal bone level can be obtained. Frequency distribution of the MBL should also be reported.

Bone levels and the thickness of the buccal plate can be evaluated by means of a CBCT examination using the appropriate technique. If a CBCT examination is included in the study protocol it should be performed at least at baseline and at the end of follow-up. From this examination the following information should be obtained:

the distance from the gingival margin to the implant shoulder (radiopaque marker) (DIM).

the thickness of the buccal and lingual walls at 2, 4 and $6 \mathrm{~mm}$ of the implant or abutment shoulders.

the percentage of cases with no detectable buccal/lingual plate, as well as the frequency distribution of the buccal/lingual bone changes.

The evaluation of the DIM could also be achieved, in four points per implant, using a clinical examination with the help of a periodontal probe, after the removal of the implant-supported prosthesis. This technique should also be considered during the follow-up, if multiple evaluations have to be made, to reduce patient exposure to radiation.

The use of optical scanners to obtain precise and non-invasive ridge and soft tissues volume information, associated with the clinical measurement of the DIM by means of a periodontal probe, could allow us to estimate the level of buccal and interproximal hard tissue, allowing us to reduce patients' exposure to radiation.

There is a need to evaluate the impact of the loss of the buccal plate on peri-implant health and its repercussions on the quality of life of the patient.

\section{The effect of zirconia or titanium as abutment material on soft tissues. A systematic review and meta-analysis}

\section{Aim}

The objective of the review was to analyse the literature with regard to the effect of zirconia or titanium as abutment materials on soft peri-implant tissues.

\section{Summary of the review}

Nine studies (11 papers) were included: four RCTs and five prospective studies (CCTs) with at least 10 patients, with a follow-up $\geq 1$ year, and with a direct comparison (splitmouth) between titanium oxide (Ti) and zirconium oxide $(\mathrm{Zr})$ abutments. Clinical parameters (PPD, BOP, REC, MBL and additional biological complications) and aesthetic outcome variables (soft tissue colour, patientreported outcomes and aesthetic indices) were analysed.

Effect of $\mathrm{Zr}$ or Ti as abutment material on soft tissues. Clinical parameters

\section{Probing pocket depth (PPD)}

PPD was recorded in three studies (five papers: Sailer et al. 2009; Zembic et al. 2009, 2012; Lops et al. 2013; Carrillo et al. 2014) and none of them showed statistically significant difference between Ti and $\mathrm{Zr}$ abutments, neither in absolute values nor in changes over time. A meta-analysis was not feasible due to heterogeneity of the reported outcomes.

\section{Bleeding on probing (BOP)}

Five studies (seven papers: Sailer et al. 2009; Zembic et al. 2009; Zembic et al. 2012; Lops et al. 2013; Hosseini et al. 2011; Hosseinni et al. 2013; Payer et al. 2012) compared bleeding on probing around $\mathrm{Zr}$ or $\mathrm{Ti}$ abutments. None of them found statistically significant differences at 1,3 and 5 years follow-up. There was also no difference in plaque indices. The latter were not considered because they were primarily assessed at the restoration rather than at the abutment level.

\section{Marginal bone level (MBL)}

Six studies (eight papers: Sailer et al. 2009; Zembic et al. 2009, 2012; Lops et al. 2013; Hosseini et al. 2011; Hosseinni et al. 2013; Payer et al. 2012; Carrillo et al. 2014) reported on interproximal marginal bone 
level changes, and did not show any statistical significant differences.

\section{Additional biological complications}

Biological complications (suppuration, swelling, fistula, implant loss) were reported in four studies (Hosseini et al. 2011; Hosseinni et al. 2013; Zembic et al. 2012; Lops et al. 2013). Three of them showed a higher frequency of biological complications at implants with Zr abutments. A meta-analysis was not meaningful due to the heterogeneity of the criteria.

\section{Soft tissue recession}

Peri-implant mucosal recession was recorded in three studies (Zembic et al. 2012; Lops et al. 2014; Carrillo et al. 2014) and again no significant differences were observed.

Effect of Zr or Ti as abutment material on soft tissues. Aesthetics

\section{Soft tissue colour}

Colour characteristics of the peri-implant mucosa were evaluated in five studies /six papers: Sailer et al. 2009; Zembic et al. 2009; Hosseini et al. 2011; Hosseinni et al. 2013; Cosgarea et al. 2014; Bressan et al. 2011), of which three used spectrophotometry and these were included in a meta-analysis. The outcome was found to be significantly superior for $\mathrm{Zr}$ abutments.

\section{Patient-reported outcome}

The evaluation by the patients themselves was reported in two studies (Hosseini et al. 2011; Carrillo et al. 2014). Both applied

\section{References}

Linkevicius, T. \& Vaitelis, J. (2015) The effect of zirconia or titanium as abutment material on soft peri-implant tissues: a systematic review and meta-analysis. Clinical Oral Implants Research 26 (Suppl. 11): 139-147. visual analogue scale (VAS). No significant difference was seen.

\section{Aesthetic indices}

The aesthetic outcomes were reported in four studies (six papers: Sailer et al. 2009; Zembic et al. 2009, 2012; Hosseini et al. 2011; Payer et al. 2012; Carrillo et al. 2014). Only one study used an index restricted to soft tissues (Pink aesthetic score) and showed a significant difference in favour of $\mathrm{Zr}$ abutments. The other three studies implemented a combined index which also included the restoration or focused on the papillae only.

\section{Consensus statement}

The literature evaluated in this systematic review revealed no significant differences in clinical outcomes in terms of PPD, BOP, MBL and mucosal recessions, when comparing zirconia and titanium as abutment materials. $\mathrm{Zr}$ abutments may be associated with more biological complications, whereas they showed significant superiority in achieving natural soft tissue colour.

\section{Clinical recommendations}

Based on the available data, both zirconia and titanium abutments have been successfully used in the anterior and posterior regions up to the premolar area of both jaws. In particular, the reported clinical outcomes (PPD, BOP, MBL and REC) were not significantly different between either material. Accordingly, the clinician may choose either $\mathrm{Zr}$ or $\mathrm{Ti}$ abutments for single-tooth restorations.

There is no clinical evidence for a reduced plaque accumulation on $\mathrm{Zr}$ abutments.
Therefore, maintenance care should be similar for both materials.

In aesthetically demanding areas, especially with thin buccal soft tissues, the use of $\mathrm{Zr}$ abutments may offer aesthetic advantages.

Some reports show a higher incidence of biological complications at implants with $\mathrm{Zr}$ abutments, however, a correlation with the abutment material could not be identified. These complications could instead be associated with submucosal cement remnants. Therefore, irrespective of the material used, the abutment must be designed with easy to access cementation margins.

\section{Implications for research}

Future studies should consider a more uniform definition of biological complications and should also report on changes, as well as absolute values, relating to primary and secondary outcomes. Radiographic assessments of MBL should be based on standardized periapical radiographs with a clear definition of the baseline reference.

The influence of the nano-, micro- and macro-structures, as well as the cleaning (chemical composition of the surface) of the trans-mucosal components should be evaluated clinically (e.g. in relation to the stability of the soft tissue).

Future studies should address indications other than single-tooth restorations.

It would be interesting to compare the aesthetic outcome of $\mathrm{Zr}$ abutments with other materials such as: colour-modified $\mathrm{Zr}$ or $\mathrm{Ti}$ abutments.
Lutz, R., Neukam, F.W. \& Schmitt, C.M. (2015) Long-term outcomes of bone augmentation on soft and hard tissue stability: a systematic review. Clinical Oral Implants Research 26 (Suppl. 11): 103-122.
Rotundo, R., Pagliaro, U., Bendinelli, E., Esposito, M. \& Buti, J. (2015) Long-term outcomes of soft tissue augmentation around dental implants on soft and hard tissue stability: a systematic review. Clinical Oral Implants Research 26 (Suppl. 11): 123-138. 